\title{
Reliability and validity of the Chinese version of the Nutritional Form For the Elderly
}

\author{
Han Gao ${ }^{1}$, Ulrika Söderhamn ${ }^{2}$, Lin Zhang ${ }^{1}$, Hui-Xia Cui ${ }^{1}$ and Kun Liu ${ }^{1, *}$ \\ ${ }^{1}$ College of Nursing, Liaoning Medical University, No. 3-40 Songpo Road, Linghe District, Jinzhou 121001, \\ Liaoning Province, People's Republic of China: ${ }^{2}$ Center for Caring Research - Southern Norway, Faculty of Health \\ and Sport Sciences, University of Agder, Grimstad, Norway
}

Submitted 18 June 2014: Final revision received 4 November 2014: Accepted 7 December 2014: First published online 20 January 2015

\begin{abstract}
Objective: The present study aimed to translate the English version of the Nutritional Form For the Elderly into Simplified Chinese, as well as to test the reliability (homogeneity and stability) and validity (content and construct validity) of the Chinese version of the Nutritional Form For the Elderly (NUFFE-CHI).

Design: The study adopted a cross-sectional design. The English version of the NUFFE was translated into Simplified Chinese and a questionnaire survey was conducted. The data were analysed with statistical methods to estimate the homogeneity, stability, content and construct validity.

Setting: Jinzhou City, China.

Subjects: A total number of 701 community-dwelling older adults answered the questionnaire, including background variables and the NUFFE-CHI. A small group of the participants ( $n$ 50) completed the NUFFE-CHI twice for test-retest reliability. Results: Cronbach's $\alpha$ was 0.65 and the split-half reliability was 0.67 . Item-to-total correlation analyses showed that the scale has sufficient internal consistency. The test-retest reliability regarding the total scores of NUFFE-CHI was reflected in an intra-class correlation coefficient of 0.88 . The intra-class correlation coefficients between the test and retest of the NUFFE-CHI items varied between 0.43 and 0.98 . A content validity index of 0.83 explained good content validity. Construct validity was demonstrated in an exploratory factor analysis with a six-factor solution, explaining $57.65 \%$ of the variance.

Conclusions: This first testing of the NUFFE-CHI indicates sufficient evidence for reliability, content and construct validity. Further testing studies regarding homogeneity, concurrent validity, sensitivity and specificity are required before the NUFFE-CHI can be used as a screening instrument in clinical settings and in research.
\end{abstract}

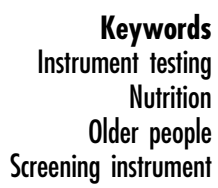

The prevalence of older people who are undernourished or at risk of undernutrition is high; for example, the prevalence rate was $27 \%$ among geriatric in- and out-patients in a French study ${ }^{(1)}$ and $22 \%$ among older home-dwelling people in a Swedish study ${ }^{(2)}$. However, among older community-dwelling Chinese people, the prevalence rate was found to be $44 \%^{(3)}$, and it was as high as $76 \%$ among very old ( $90+$ years) Chinese community-dwelling people ${ }^{(4)}$. In other Chinese studies performed among hospitalised patients, the prevalence has been found to vary between $20 \%$ and $40 \%{ }^{(5,6)}$.

To be undernourished or at risk of undernutrition among older people is an extensive problem, since it is associated with several serious factors including morbidity, longer hospital stays, mortality, deteriorated functional ability $^{(7)}$, mobility impairment ${ }^{(8)}$, cognitive dysfunction ${ }^{(4,7,8)}$, reduced well-being ${ }^{(7)}$ and poor self-rated health ${ }^{(2,4)}$. Moreover, older people who are widowed $^{(3)}$, living alone ${ }^{(2,9)}$ and of advanced age $\mathrm{e}^{(3,4,8)}$ are especially vulnerable for being at risk for undernutrition.

In order to find potential and existing nutritional problems in community-dwelling older adults, and in order to prevent the occurrence and aggravation of individuals' nutritional status and its associated negative consequences, we need a screening instrument. Therefore, there is a need for a simple screening instrument for use among older people that can be used by both the older persons themselves and health professionals. The nutritional screening instrument, the Nutritional Form For the Elderly (NUFFE), which was originally developed in Sweden by Söderhamn 
and Söderhamn ${ }^{(10,11)}$, is a simple and useful instrument for identifying the risk of undernutrition in older adults ${ }^{(10,11)}$.

The main features of NUFFE are that: (i) it is specially designed for older people; (ii) it does not include any anthropometric measurements or complex calculations and its items are easy to operate (they can even be filled out by the older adult, him- or herself); and (iii) it is relatively short and is usually completed in $5-10 \mathrm{~min}^{(12)}$. NUFFE has been translated into English, Hungarian, Norwegian, German and Italian ${ }^{(12)}$. The Swedish ${ }^{(10,11)}$, Norwegian ${ }^{(13)}$ and Hungarian versions ${ }^{(14)}$ of NUFFE have been tested in terms of their reliability and validity among older hospital patients, and the Norwegian version ${ }^{(15,16)}$ has also been tested among older home-dwelling people. The results have shown sufficient evidence for the reliability and validity of these versions in order to be used as a screening instrument ${ }^{(10,11,13-16)}$. However, there is no Chinese version, so there is a need to translate NUFFE into Simplified Chinese and to test its reliability and validity, so as to obtain a convenient nutritional screening instrument.

Therefore, the aim of the present study was to translate the English version of the NUFFE into Simplified Chinese, as well as to test the reliability (homogeneity and stability) and validity (content and construct validity) of the Chinese version of NUFFE (NUFFE-CHI).

\section{Methods}

\section{Design and participants}

The present study adopted a cross-sectional design and was carried out in the spring and summer of 2013 in Jinzhou City, China. Sixteen questionnaire survey stations were set in eight communities in three districts. We publicised the study to people in these communities, and they came to the survey stations voluntarily and provided their written informed consent before completing the questionnaire. The inclusion criteria used for the participants were as follows: (i) $\geq 60$ years of age; (ii) able to communicate; and (iii) provided consent to participate. Finally, a number of 701 elderly persons completed the questionnaires. The City of Jinzhou has a population of about 3 million and an estimated elderly population (>60 years) of 450000 people $^{(17)}$. A sample size of $n 701$ is assumed to be sufficient because post hoc calculations of the questionnaire's margin of error with a sample size of $n 701$ gave a margin of error of $1.6 \%$ with a confidence interval of $95 \%$. This margin of error level is regarded as acceptable $^{(18)}$.

\section{The translation procedure}

The English version of NUFFE was provided by one of its original authors for the convenience of translation. Following Brislin's ${ }^{(19)}$ translation guidelines, several translation procedures were undertaken. First, two bilingual professional translators translated the NUFFE from English into Simplified
Chinese. Another two bilingual professional translators translated the Chinese version back into English. Second, a bilingual expert panel consisting of two nursing postgraduates, three nursing staff members and two nursing teachers evaluated the cultural and linguistic equivalence of each item. Third, the Chinese version was presented to ten elderly people and a modification was made according to the participants' degree of understanding, as well as in response to the participants' feedback on the instrument. Finally, we achieved the Chinese version of the NUFFE after a consensus was reached in terms of its wording, clarity and cultural equivalence.

\section{Data collection}

The questionnaire that was used consisted of the Chinese version of the NUFFE, as well as background variables such as age, sex, marital status and diseases. The older adults were interviewed face to face by trained interviewers who were postgraduates from a nursing college. Prior to data collection, the interviewers received unified training to learn how to ask and explain the items in the same way. Fifty participants completed the NUFFE-CHI a second time via telephone calls two weeks after the initial data collection in order to provide data for test-retest reliability.

\section{The instrument}

The NUFFE consists of a summated, three-point ordinal scale with fifteen items concerning weight loss, changes in dietary intake, appetite, food and liquid intake, eating difficulties, the ability to obtain food products, one's company at meals, activity levels and the number of prescription drugs being taken. The response alternatives for each item ranged between zero and two. The most favourable option yielded a score of 0 and the most unfavourable option resulted in a score of 2 . The intermediate option provided a score of 1 . The maximum score total is 30 , where a higher score indicates a higher degree of risk for undernutrition ${ }^{(12)}$.

\section{Statistical analysis}

Reliability was estimated as homogeneity by calculating item-to-total correlations and Cronbach's $\alpha$ coefficient. The item-to-total correlations were calculated by Spearman rank correlations (two-tailed probability) between each item and the scale total of the NUFFE-CHI when the particular item was omitted from the scale ${ }^{(20)}$. In addition to Cronbach's $\alpha$ coefficient for the total score, split-half reliability was assessed with the Cronbach's $\alpha$ coefficient of the scores of two groups: i.e. a parity bisection method divided the entries into two groups according to the sequence numbers and Cronbach's $\alpha$ coefficients were calculated, respectively.

Reliability was also estimated as stability by calculating two-way mixed intra-class correlation coefficients (ICC) for absolute agreement with $95 \%$ confidence intervals ${ }^{(20)}$ 
from the fifty participants' two total NUFFE-CHI scores and their item scores in order to obtain test-retest reliability.

In order to estimate content validity, five experts were invited to evaluate the items of the NUFFE-CHI. Every expert gave a mark to each item according to four grades: $1=$ not related; $2=$ weak correlation; $3=$ strong correlation; and $4=$ very relevant. The 'not related' and 'weak correlation' options yielded a score of 0 , and the 'strong correlation' and 'very relevant' options yielded a score of 1 . The content validity index (CVI) was estimated by dividing the sum of the index of the five experts by the number of experts. A CVI by five experts that was greater than 0.80 showed good content validity ${ }^{(21)}$.

The construct validity was estimated by an exploratory factor analysis (with orthogonal rotation - Varimax with Kaiser normalisation) in order to explore the model of the NUFFE-CHI.

All analyses were conducted using the statistical software package IBM SPSS Statistics 17·0.

\section{Ethical considerations}

The study was conducted according to the guidelines presented in the Declaration of Helsinki ${ }^{(22)}$. The Research Ethics Committee, Liaoning Medical University, Jinzhou, China, approved all procedures involving human subjects. Written informed consent was obtained from all participants. The questionnaires were filled out voluntarily and without names. Throughout the entire research process, the participants' privacy was kept strictly confidential.

\section{Results}

\section{The sample}

Of the participating 701 older adults, 313 (44.7\%) were men and 388 (55.3\%) were women. Their ages ranged between 60 and 98 years, with a mean age of $69 \cdot 18$ (sD 7.94) years old. Six hundred and forty-nine individuals were married $(92.6 \%)$, eleven were unmarried (1.6\%), fourteen were divorced $(2.0 \%)$, twenty-seven were remarried $(3.9 \%)$ and $140(20.0 \%)$ were bereft of spouse. The most common diseases were hypertension (28.8\%), CHD (17.1\%), rheumatoid arthritis (16.4\%), diabetes (12.7\%) and gastrointestinal tract disease (9.3\%).

\section{Reliability}

The homogeneity of the NUFFE-CHI, as a measure of reliability, was shown with a Cronbach's $\alpha$ coefficient of 0.65 and split-half reliability of $0 \cdot 67$. The homogeneity was also reflected in statistically significant $(P<0.001)$ item-to-total score correlations (Table 1).

The stability of NUFFE-CHI, as a measure of reliability, was reflected in the ICC of 0.88 (95\% CI $0.80,0.93 ; P<0.001$ ) between the two total scores (test-retest) of the NUFFE-CHI obtained. The ICC obtained between the two NUFFE-CHI item scores in the test-retest are presented in Table 2.
Table 1 Item-to-total score correlations (Spearman rank) for the Chinese version of the Nutritional Form For the Elderly (NUFFE-CHI) (n 701)

\begin{tabular}{clc}
\hline Item no. & Item content & $r_{\mathrm{s}}$ \\
\hline 1. & Weight loss & 0.35 \\
2. & Changes in dietary intake & 0.46 \\
3. & Appetite & 0.44 \\
4. & Intake of cooked food & 0.18 \\
5. & Portion size & 0.47 \\
6. & Intake of fruit and vegetables & 0.32 \\
7. & Possibility of obtaining food products & 0.45 \\
8. & Company at meals & 0.47 \\
9. & Activity & 0.26 \\
10. & Tooth/mouth and swallowing difficulties & 0.39 \\
11. & Fluid intake & 0.44 \\
12. & Gastrointestinal problems & 0.43 \\
13. & Help with eating & 0.13 \\
14. & Number of drugs & 0.42 \\
15. & Health state & 0.37 \\
\hline
\end{tabular}

\section{Validity}

The CVI assessed by the five experts was $0 \cdot 83$, which served as a measure of content validity.

A Kaiser-Meyer-Olkin value of 0.729 and a Bartlett spherical test value of $1113.150(P<0.001$ at the 0.01 test level) in an exploratory factor analysis of the fifteen entries showed that the factor analysis was feasible. The method extracted six common factors where the eigenvalues were greater than 1 after the Varimax orthogonal rotation. A sixfactor solution explained $57.65 \%$ of the variance. Fifteen of the entries' factor loadings and communalities are displayed in Table 3 .

\section{Discussion}

Since the prevalence of undernourished Chinese older

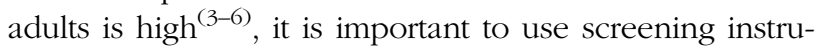
ments to identify older people who are at risk for undernutrition. The NUFFE is a simple and useful screening instrument that has been translated into several languages for determining the risk of undernutrition among older adults $^{(10-12)}$. The present research is the first attempt to translate the NUFFE into Simplified Chinese and to test the NUFFE-CHI among older Chinese people. Testing of this measure showed sufficient evidence of homogeneity and stability, as well as of content and construct validity. Therefore, the NUFFE-CHI might be conducive for use among older adults in order to improve the nutritional status of community-dwelling Chinese older adults.

The homogeneity or internal consistency of the NUFFECHI was evaluated by Cronbach's $\alpha$ coefficient, split-half reliability and item-to-total score correlations, and the stability of the instrument was evaluated by the test-retest calculation of ICC. The Cronbach's $\alpha$ coefficient of 0.65 and the split-half reliability of 0.67 were assessed as being satisfactory. However, a Cronbach's $\alpha$ coefficient between 0.70 and 0.90 is the recommended value for an instrument 
Table 2 Agreement on items between the test and retest of the Chinese version of the Nutritional Form For the Elderly (NUFFE-CHI) using intra-class correlation coefficients $(\mathrm{ICC})^{*}$ with $95 \%$ confidence intervals $(n 50)$

\begin{tabular}{lllr}
\hline Items & ICC & $95 \% \mathrm{Cl}$ & $P$ value \\
\hline 1. Weight loss & 0.65 & $0.46,0.79$ & $<0.001$ \\
2. Changes in dietary intake & 0.51 & $0.27,0.69$ & $<0.001$ \\
3. Appetite & 0.67 & $0.49,0.80$ & $<0.001$ \\
4. Intake of cooked food & 0.66 & $0.47,0.79$ & $<0.001$ \\
5. Portion size & 0.43 & $0.23,0.66$ & $<0.001$ \\
6. Intake of fruit and vegetables & 0.63 & $0.43,0.77$ & $<0.001$ \\
7. Possibility of obtaining food products & 0.67 & $0.48,0.80$ & $<0.001$ \\
8. Company at meals & 0.98 & $0.96,0.99$ & $<0.001$ \\
9. Activity & 0.55 & $0.32,0.72$ & $<0.001$ \\
10. Tooth/mouth and swallowing difficulties & 0.46 & $0.21,0.65$ & $<0.001$ \\
11. Fluid intake & 0.93 & $0.87,0.96$ & $<0.001$ \\
12. Gastrointestinal problems & 0.44 & $0.19,0.64$ & $<0.001$ \\
13. Help with eating & 0.66 & $0.47,0.79$ & $<0.001$ \\
14. Number of drugs & 0.93 & $0.89,0.96$ & $<0.001$ \\
15. Health state & 0.65 & $0.46,0.79$ & $<0.001$ \\
\hline
\end{tabular}

*Two-way mixed ICC for absolute agreement.

Table 3 Exploratory factor analysis of the Chinese version of the Nutritional Form For the Elderly (NUFFE-CHI) $(n$ 701)

\begin{tabular}{|c|c|c|c|c|c|c|c|}
\hline Items & $\mathrm{F} 1$ & $\mathrm{~F} 2$ & F3 & $\mathrm{F} 4$ & F5 & F6 & Communality \\
\hline 2. Changes in dietary intake & 0.734 & & & & & & 0.645 \\
\hline 3. Appetite & 0.656 & & & & & & 0.576 \\
\hline 5. Portion size & 0.654 & & & & & & 0.570 \\
\hline 1. Weight loss & 0.643 & & & & & & 0.536 \\
\hline 12. Gastrointestinal problems & & 0.790 & & & & & 0.668 \\
\hline 10. Tooth/mouth and swallowing difficulties & & 0.698 & & & & & 0.528 \\
\hline 15. Health state & & 0.698 & & & & & 0.528 \\
\hline 8. Company at meals & & & 0.795 & & & & 0.658 \\
\hline 7. Possibility of obtaining food products & & & 0.653 & & & & 0.484 \\
\hline 6. Intake of fruit and vegetables & & & & 0.654 & & & 0.613 \\
\hline 13. Help with eating & & & & 0.654 & & & 0.580 \\
\hline 11. Fluid intake & & & & & 0.800 & & 0.660 \\
\hline 14. Number of drugs & & & & & & 0.685 & 0.580 \\
\hline 9. Activity & & & & & 0.429 & 0.608 & 0.625 \\
\hline 4. Intake of cooked food & & & & & & -0.406 & 0.397 \\
\hline
\end{tabular}

Solution with six factors (F1 to F6) is shown. Only factor loadings with an absolute value $>0.40$ are displayed.

according to Streiner and Norman ${ }^{(20)}$, but the authors also mean that if an instrument consists of causal indicators, it is not crucial that the instrument has a high degree of homogeneity. The reason for this statement is that causal indicators define the underlying construct by their presence ${ }^{(20)}$. The NUFFE consists of risk factors for undernutrition that can be regarded as causal indicators; thus, a Cronbach's $\alpha$ coefficient of 0.65 is determined to be satisfactory. Corresponding Cronbach's $\alpha$ coefficients of $0.72,0.70,0.77,0.71,0.64$ and 0.62 have been obtained in the Swedish ${ }^{(10,11)}$, Norwegian ${ }^{(13,15,16)}$ and Hungarian ${ }^{(14)}$ testing studies of NUFFE, respectively.

All item-to-total correlations were statistically significant; however, the correlation coefficients of items 4 and 13 were less than $0 \cdot 20$. The low correlation of these two items may be due to the fact that most of the participants used to eat cooked food every day and they did not need help to eat. One possible explanation for the low values can, therefore, be that the sample was rather homogeneous. In order to test an instrument's homogeneity, it is crucial that a heterogeneous sample is obtained, which will guarantee that all degrees of nutritional status are represented and thus all response alternatives of the items will be used. It can be argued that items with correlation coefficients below 0.20 should be deleted. However, since these two items are associated with the older adults' nutrition, these items might contribute to measuring the risk of undernutrition, although there were low correlations in the present study ${ }^{(20)}$. A step-by-step analysis of the items found that deleting any item could reduce the Cronbach's $\alpha$ coefficient ${ }^{(23)}$, so this could also be a reason why items 4 and 13 should not be deleted. However, in testing studies of the Swedish ${ }^{(10,11)}$ and Hungarian versions of the NUFFE ${ }^{(14)}$, the same items had a low correlation with the total scale. In the Norwegian testing studies ${ }^{(13,15,16)}$, only item 13 had a low correlation with the total scale. These results indicate, however, that item 13 does not contribute to measuring the risk for undernutrition; this may be due to the fact that older adults can manage to eat by themselves. 
The ICC of 0.88 obtained for the test-retest of the two total NUFFE-CHI scores showed very good stability. The agreement between the test and retest across items showed that five items had moderate agreement, seven items had substantial agreement and three items had almost perfect agreement, according to Landis and $\mathrm{Koch}^{(24)}$ and $\mathrm{Zou}^{(25)}$, who indicated that an ICC in the range of $0 \cdot 00-0 \cdot 20$ represents slight agreement; $0 \cdot 21-0 \cdot 40$ represents fair agreement; $0 \cdot 41-0.60$ represents moderate agreement; 0.61-0.80 represents substantial agreement; and 0.81-1.00 represents almost perfect agreement. A rather similar agreement was obtained in a test-retest of the Norwegian version of the NUFFE using the weighted $\kappa^{(13)}$.

The CVI of 0.83 indicated good content validity ${ }^{(21)}$. The construct validity of the NUFFE-CI was estimated with an exploratory factor analysis in the present study. Construct validity is normally evaluated by factor analysis; ideal factor analysis suggests that each item should have a higher load value $(>0.40)$ on one of the common factors, while having low load values on other common factors, and that the cumulative variance contribution ratio of the common factors is at least $40 \%{ }^{(23)}$. The exploratory factor analysis model of the NUFFE-CHI had six common factors, which was consistent with the number of common factors for the Swedish version. The items for factor 1 were almost the same as in the testing studies of the Swedish version ${ }^{(11)}$ and the Hungarian version ${ }^{(14)}$, except that item 15 , 'Health state', did not appear in factor 1 in our study. This might be because most of the participants in our study felt that they were healthy. Factor 5 had the same items as the Swedish version $^{(11)}$. The differences in the other factors might be due to the participants' different cultural background. Table 3 shows that the load value for each item was greater than 0.40 . The communality of each item ranged from 0.397 to $0 \cdot 668$, and the total explained variance was $57 \cdot 65 \%$, which indicated acceptable construct validity.

\section{Limitations}

One limitation of the present study is that the English version of the NUFFE was used to translate the NUFFE into Simplified Chinese. The most optimal situation would have been to translate the original Swedish version of the NUFFE. However, the Swedish version of the NUFFE has been translated into English by professional translators according to the procedure recommended by Streiner and Norman $^{(20)}$.

Another limitation is that a small number of participants answered the NUFFE-CHI a second time in order to estimate the stability of the instrument. Moreover, these data were not collected using the same methods during the test-retest procedure. The use of telephone interviews during the retest procedure, rather than face-to-face interviews, can be assumed to weaken the results.

It is a challenge to include the oldest old in research studies ${ }^{(26)}$. The low mean age in the present study indicates that few very older adults were included. Advanced age is known to be associated with being at risk for undernutrition or with being undernourished ${ }^{(3,4,8)}$. With more very old individuals in the sample, it can be assumed that the population would be increasingly heterogeneous, which is desirable for a testing study. Therefore, it would have been preferable that another recruitment process was used. For example, it can be assumed that a randomised sample would result in a more heterogeneous sample.

Furthermore, it was not possible to estimate concurrent validity, sensitivity and specificity in order to determine an appropriate cut-off value for identifying older people at risk for undernutrition, as no other nutritional instrument was included in the questionnaire as a gold standard.

Despite these limitations, the results of the reliability and validity tests of the NUFFE-CHI have similarities with earlier testing studies of the NUFFE ${ }^{(10,11,13-16)}$; as such, this strengthens the testing results of the NUFFE-CHI.

\section{Conclusion}

The present study offers the first testing of the NUFFE-CHI in terms of its homogeneity and stability, as well as of its content and construct validity. This initial testing indicates that the NUFFE-CHI provided sufficient evidence as a reliable and valid screening instrument. However, further testing studies among a more heterogeneous sample, so as to estimate homogeneity, concurrent validity, sensitivity and specificity, are required before the NUFFE-CHI can be used as a screening instrument in clinical settings and in research.

\section{Acknowledgements}

Acknowledgements: The authors are grateful to Fang Chen, Siyi Du, Qian Su and Lili Zhang, who gave selfless support of the study, and thank Yu Gao and her colleagues for their help to translate the instrument. English-language editing of this manuscript was provided by Journal Prep. Financial support: This research received no specific grant from any funding agency in the public, commercial or not-for-profit sectors. Conflict of interest: None. Authorship: K.L. and H.G. conceived and designed the experiments. H.G., L.Z. and H.X.C. performed the data collection. H.G. and U.S. analysed the data. H.G. and U.S. wrote the manuscript. L.Z., H.X.C. and K.L. reviewed the manuscript. All authors approved the final version. Ethics of buman subject participation: The study was conducted according to the guidelines presented in the Declaration of Helsinki and all procedures involving human subjects were approved by the Research Ethics Committee, Liaoning Medical University, Jinzhou, China. Written informed consent was obtained from all participants, the questionnaires were filled out voluntarily and without names, and the participants' privacy was kept strictly confidential throughout the entire research process. 


\section{References}

1. Rolland Y, Perrin A, Gardette V et al. (2012) Screening older people at risk of malnutrition or malnourished using the Simplified Nutritional Appetite Questionnaire (SNAQ): a comparison with the Mini-Nutritional Assessment (MNA) tool. J Am Med Dir Assoc 13, 31-34.

2. Söderhamn U, Christensson L, Idvall E et al. (2012) Factors associated with nutritional risk in 75-year-old community living people. Int J Older People Nurs 7, 3-10.

3. Han Y, Li S \& Zheng Y (2009) Predictors of nutritional status among community-dwelling older adults in Wuhan, China. Public Health Nutr 12, 1189-1196.

4. Ji L, Meng H \& Dong B (2012) Factors associated with poor nutritional status among the oldest-old. Clin Nutr 31, 922-926.

5. Fang S, Long J, Tan R et al. (2013) A multicentre assessment of malnutrition, nutritional risk, and application of nutritional support among hospitalized patients in Guangzhou hospitals. Asia Pac J Clin Nutr 22, 54-59.

6. Zhang H, Wang Y, Mu SY et al. (2012) Prevalence of nutritional risks, malnutrition and application of nutritional support rates at one Chongqing teaching hospital. Zhonghua Yi Xue Za Zhi 92, 3417-3419 (in Chinese).

7. Torma J, Winblad U, Cederholm $\mathrm{T}$ et al. (2013) Does undernutrition still prevail among nursing home residents? Clin Nutr 32, 562-568.

8. Hirose T, Hasegawa J, Izawa S et al. (2014) Accumulation of geriatric conditions is associated with poor nutritional status in dependent older people living in the community and in nursing homes. Geriatr Gerontol Int 14, 198-205.

9. Söderhamn U, Dale B, Sundsli K et al. (2012) Nutritional screening of older home-dwelling Norwegians: a comparison between two instruments. Clin Interv Aging 7, 383-391.

10. Söderhamn U \& Söderhamn O (2001) Developing and testing the Nutritional Form For the Elderly. Int J Nurs Pract 7, 336-341.

11. Söderhamn U \& Söderhamn O (2002) Reliability and validity of the nutritional form for the elderly (NUFFE). $J$ Adv Nurs 37, 28-34.

12. Söderhamn U (2011) The Nutritional Form For the Elderly (NUFFE) - a short scale development report. Nordisk Sygeplejeforskning/Nordic Nurs Res 1, 75-81.
13. Söderhamn U, Flateland S, Jessen L et al. (2009) Norwegian version of the Nutritional Form for the Elderly: sufficient psychometric properties for performing institutional screening of elderly patients. Nutr Res 29, 761-767.

14. Gombos T, Kertesz K, Csikos A et al. (2008) Nutritional form for the elderly is a reliable and valid instrument for the determination of undernutrition risk, and it is associated with health-related quality of life. Nutr Res 28, 59-65.

15. Söderhamn U, Dale B, Sundsli K et al. (2012) Psychometric testing of the Norwegian version of the Nutritional Form For the Elderly among older home-dwelling people. J Multidiscip Healthc 5, 121-128.

16. Tomstad ST, Söderhamn U, Espnes GA et al. (2013) Testing two self-care-related instruments among older home-dwelling people in Norway. Int J Older People Nurs 8, 189-198.

17. Chen F \& Liu G (2009) Population ageing in China. In International Handbook of Population Ageing, pp. 157-172. [P Uhlenberg, editor]. Dordrecht: Springer.

18. Groves RM, Fowler FJ, Couper MP et al. (2009) Survey Methodology, 2nd ed. Hoboken, NJ: John Wiley \& Sons, Inc.

19. Brislin RW (1970) Back-translation for cross-cultural research. J Cross Cult Psychol 1, 185-216.

20. Streiner DL \& Norman GR (2008) Health Measurement Scales - A Practical Guide to Their Development and Use, 4th ed. Oxford: Oxford University Press.

21. Shi J, Mo X \& Sun Z (2012) Content validity index in scale development. Zhong Nan Da Xue Xue Bao Yi Xue Ban 37, 152-155 (in Chinese).

22. Declaration of Helsinki (2013) Ethical Principles for Medical Research Involving Human Subjects. Fortaleza, Brazil: World Medical Association; available at http://www.wma. net/en/30publications/10policies/b3/index.html

23. Liu K (2012) The Application of SPSS in Medical Scientific Research. Beijing: People's Medical Publishing House (in Chinese).

24. Landis JR \& Koch GG (1977) The measurement of observer agreement for categorical data. Biometrics 33, 159-174.

25. Zou GY (2012) Sample size formulas for estimating intraclass correlation coefficients with precision and assurance. Stat Med 31, 3972-3981.

26. Jacelon CS (2007) Older adults' participation in research. Nurs Res 14, 64-73. 